\title{
Comparação da força muscular e flexibilidade entre praticantes de Pilates e musculação
}

\author{
Comparison of muscle strength and flexibility \\ between Pilates and bodybuilding
}

\author{
Altair Argentino Pereira Junior ${ }^{1}$ (D) \\ Andréia da Silva² ${ }^{\mathbb{D}}$
}

${ }^{1}$ Autor para correspondência. Centro Universitário de Brusque (Brusque). Santa Catarina, Brasil. altjunior@unifebe.edu.br ${ }^{2}$ Fisioterapeuta (Brusque). Santa Catarina, Brasil. andreia_brq@hotmail.com

\begin{abstract}
RESUMO | INTRODUÇÃo: Nos últimos anos, tem aumentado o número de estudos preocupados em investigar a relação entre a disfunção do músculo transverso do abdome e a história de dor lombar, assim como a deficiência na flexibilidade. OBJETIVO: comparar a força muscular do transverso do abdome e flexibilidade das praticantes do método Pilates e musculação. MÉTODO: Participaram do estudo 20 voluntárias do sexo feminino divididas em 2 grupos de 10 voluntárias, com no mínimo 6 meses de atividade em Pilates ou musculação. A força muscular do transverso do abdome foi avaliada pelo teste esfigmomanômetro modificado (TEM) e a flexibilidade pelo teste de sentar-alcançar (TSA). Foi utilizado a análise estatística descritiva para os dados coletados, sendo os valores expressos através da média e desvio padrão, bem como aplicabilidade do teste T de student $(p<0,05)$. RESULTADOS: Foi verificado valores significativos de $(p<0,05)$ sendo superior para a força muscular do transverso do abdome do grupo Pilates quando comparado ao grupo musculação. Entretanto para o teste de flexibilidade, não foi encontrado diferenças estatísticas significativas entre os dois grupos. CONCLUSÃO: Foi possível observar que as praticantes do método Pilates obtiveram um meIhor desempenho da musculatura do transverso do abdome e flexibilidade em comparação ao grupo musculação. O fortalecimento do músculo transverso do abdome bem como a flexibilidade torna-se uma medida corretiva e preventiva para redução e prevenção das algias e lesões lombares.
\end{abstract}

PALAVRAS-CHAVE: Força muscular. Flexibilidade. Modalidades de fisioterapia.

\begin{abstract}
BACKGROUND: In recent years, it has increased the number of studies concerned with investigating the relationship between the dysfunction of transverse abdominal muscle and the history of lower back pain, as well as the deficiency in flexibility. OBJECTIVE: to compare the muscular strength of the transverse abdomen and flexibility of Pilates and and bodybuilding. METHOD: The study included 20 female volunteers divided into 210 voluntary groups, with at least 6 months of activity in Pilates or bodybuilding. Muscle strength abdomen transversus was evaluated by modified sphygmomanometer test (TEM) and flexibility the sit-reach test (TSA). We used descriptive statistical analysis to the data collected, and the amounts are expressed as mean and standard deviation as well as applicability of test $T$ student ( $p$ $<0.05)$. RESULTS: It was found significant values of $(p<0.05)$ being superior to muscle strength Pilates group of the transversus abdominis when compared to the bodybuilding. However, for the flexibility test, it was not found statistically significant differences between the two groups. CONCLUSION: It was observed that the Pilates practitioners obtained a better performance the abdomen transversus muscle and flexibility compared to the bodybuilding. The strengthening of the transverse abdominal muscle and flexibility becomes a corrective and preventive measure to reduce and prevent pains and back injuries.
\end{abstract}

KEYWORDS: Muscle Strength. Flexibility. Physical therapy modalities. 


\section{Introdução}

$\mathrm{Na}$ atualidade, a comunidade científica tem dedicado considerável destaque a discussão sobre a relação entre exercícios físicos, condições de saúde e qualidade de vida e os malefícios surgidos a partir do estado de sedentarismo ${ }^{1 \underline{2}}$. Esse debate tem levado grande parte dos profissionais de saúde a considerar os exercícios físicos um importante aliado para uma condição de vida e envelhecimento saudáveis ${ }^{3}$.

Já na circunstancia de prevenção, a força muscular, estabilidade muscular e a flexibilidade são parâmetros fisiológicos relacionados com a saúde e aptidão física. É fato que o estado de sedentarismo pode afetar a saúde em geral de mulheres se não treinadas, aumentando o risco de lesões, e diminuindo o desempenho das atividades de vida diária.

A estabilidade da coluna vertebral é garantida pela atuação de componentes ativos (músculos e tendões), passivos (ossos, discos intervertebrais, ligamentos e fáscias) e da unidade de controle neural ${ }^{5}$. Em destaque, o transverso do abdome possui fibras em orientação transversa originando-se dos processos transversos das vértebras lombares via fáscia tóraco-lombar e inserindo anteriormente na linha alba. A contração deste músculo resulta em aumento da tensão da fáscia tóraco-lombar e acréscimo da pressão intra-abdominal pela depressão da parede do abdome. Logo, o transverso do abdome contribui para a estabilização lombar por estes dois mecanismos ${ }^{6}$.

No que se refere a flexibilidade é a habilidade de mover uma ou várias articulações de maneira suave confortável por meio da amplitude de movimento irrestrita e sem dor ${ }^{7}$. Estudos apontam que a boa flexibilidade contribui para a melhor qualidade de vida do paciente, auxilia na reeducação postural e pode prevenir as lesões musculoesqueléticas ${ }^{8,9}$.

Diante disto, existe uma preocupação maior por parte dos Fisioterapeutas, demais profissionais de saúde e da sociedade em geral em reconhecer os benefícios da prática do método Pilates, na qual vem crescendo e se popularizando, destacando os benefícios conquistados por praticantes de todas as idades.
O Pilates visa o condicionamento físico e mental, contribuindo para o ganho de força, alongamento, flexibilidade, equilíbrio, preocupando-se em manter as curvaturas fisiológicas do corpo e tendo o abdome como centro de força, o qual trabalha constantemente em todos os exercícios da técnica. Neste contexto, a maioria dos ensaios clínicos nos últimos cinco anos sobre o uso do Pilates como ferramenta de reabilitação constatou que ele é eficaz na obtenção dos resultados desejados, particularmente para a redução da dor e incapacidade ${ }^{10}$.

Da mesma forma, os exercícios resistidos também chamados de exercícios de força ou musculação é uma modalidade que apresenta numerosos benefícios neuromusculares e fisiológicos, bem como sociais e comportamentais. Através da prática regular, o aumento da massa magra proporciona aos seus praticantes maior resistência e força muscular, além de maior mobilidade ${ }^{11}$.

Diante do exposto, este trabalho teve como objetivo comparar a força muscular do transverso do abdome e flexibilidade das praticantes do método Pilates e musculação.

\section{Materiais e Métodos}

Trata-se de uma pesquisa de caráter quantitativo, comparativo e transversal. Participaram desse estudo 20 mulheres na faixa etária que compreende dos 30 a 45 anos de idade, residentes na cidade de Brusque-SC distribuídas em dois grupos:

- Grupo de praticantes de Pilates composto por 10 mulheres.

- Grupo de praticantes de musculação composto por 10 mulheres.

As mesmas foram recrutadas em um estúdio de Pilates e em uma academia, respectivamente, localizados na cidade de Brusque/SC. Todas as voluntárias foram informadas quanto aos procedimentos e objetivos do estudo e após concordarem, assinaram um termo de consentimento livre e esclarecido. Os procedimentos aplicados foram aprovados pelo Comitê de Ética em Pesquisa (CAAE: 55811316.3.0000.5636) do Centro Universitário de Brusque - Unifebe. 
Os critérios de inclusão foram: praticar exclusivamente o método Pilates ou a musculação por um período no mínimo de seis meses e que apresentassem condições clínicas para realizarem os testes. Os critérios de exclusão foram: gestantes, diagnóstico de patologias ortopédicas, reumáticas ou neurológicas ou com algum comprometimento cognitivo que as impossibilitassem de compreender o desenvolvimento do estudo.

A coleta de dados foi realizada por meio de aplicação do teste do esfigmomanômetro modificado (TEM) para mensurar a força muscular do transverso do abdome. O TEM envolve o esfigmomanômetro aneroide, equipamento de baixo custo, de fácil aplicação e fornece valores objetivos que podem ser associados a medidas de força muscular. A confiabilidade e validade do TEM já foram analisadas em algumas populações, sendo encontrados resultados apropriados ${ }^{12}$. Para mensurar a flexibilidade de coluna vertebral e posteriores de coxa, foi aplicado o teste de sentar-alcançar (TSA) proposto originalmente por Wells e Dillon em 1952, segundo a padronização do Canadian Staandardized Test of Fitness (CSTF, 1986). Sendo um dos mais conhecidos e bem difundidos métodos para avaliar a flexibilidade em centros de treinamento e academias, pelo fato de ser um teste de fácil aplicação, baixo custo e padronizado ${ }^{13}$.

Para avaliar a ativação do transverso do abdome foi utilizado o esfigmomanômetro como unidade de biofeedback pressórico, da marca SOLIDOR ${ }^{\circledR}$. A avaliada sendo posicionada em supino, foi ensinada a ativar a musculatura profunda abdominal com instruções verbais, como encolher o abdome levando o umbigo em direção à coluna no tempo expiratório e tentar mantê-la, enquanto encolhia suavemente e deprimia os músculos abdominais. Com o dispositivo inflado a uma pressão inicial de $40 \mathrm{mmHg}$ na região umbilical. Quanto mais eficaz a contração, maior seria a redução de pressão. Durante as avaliações foi realizado um controle para que não houvesse compensações com outros grupos musculares, como glúteos, quadríceps e outros músculos lombares. A posição adotada a posição em decúbito ventral para a realização do teste, pois é a melhor forma de se realizar a contração correta do transverso do abdômen, diminuindo assim as compensações de outros grupos musculares durante o exercício ${ }^{14}$. O resultado esperado foi que após a contração a pressão caísse de 4 a $10 \mathrm{mmHg}$ e que fosse mantida por 10 segundos. Neste caso, a ativação era considerada como satisfatória. Caso a diferença de pressão gerada ou o tempo não estivesse dentro destes parâmetros, a ativação era considerada insatisfatória.

No TSA foi realizado com o instrumento chamado Banco de Weels. Trata-se de uma caixa medindo 30,5 $\mathrm{cm} \times 30,5 \mathrm{~cm} \times 30,5 \mathrm{~cm}$ com uma escala de $58,0 \mathrm{~cm}$ em seu prolongamento, da marca CARDIOMED $®$ sendo que o ponto zero se encontra na extremidade mais próxima do avaliado e o $26^{\circ} \mathrm{cm}$ coincide com o ponto de apoio dos pés. A avaliada retira o calçado e na posição sentada deveria tocar os pés na caixa com os joeIhos estendidos. Com ombros flexionados, cotovelos estendidos e mãos sobrepostas executou a flexão do tronco à frente devendo esta tocar o ponto máximo da escala com as mãos. Foram realizadas três tentativas sendo considerada apenas a melhor marca.

Para uma maior credibilidade do estudo apresentado, foi realizado um estudo estatístico de resultados obtidos quanto a força muscular do transverso do abdome e flexibilidade das participantes. Foram calculados por meio de estatística descritiva e os valores foram expressos através de média e desvio padrão da média, bem como aplicabilidade do teste T de student com nível de significância adotado de $a=0,05$ para detectar a diferença da força muscular e flexibilidade entre os grupos.

\section{Resultados}

Na figura 1, representa os dados coletados do teste do esfigmomanômetro modificado, avaliando a força muscular do transverso do abdome em $\mathrm{mmHg}$ entre as praticantes do grupo Pilates e grupo musculação. 


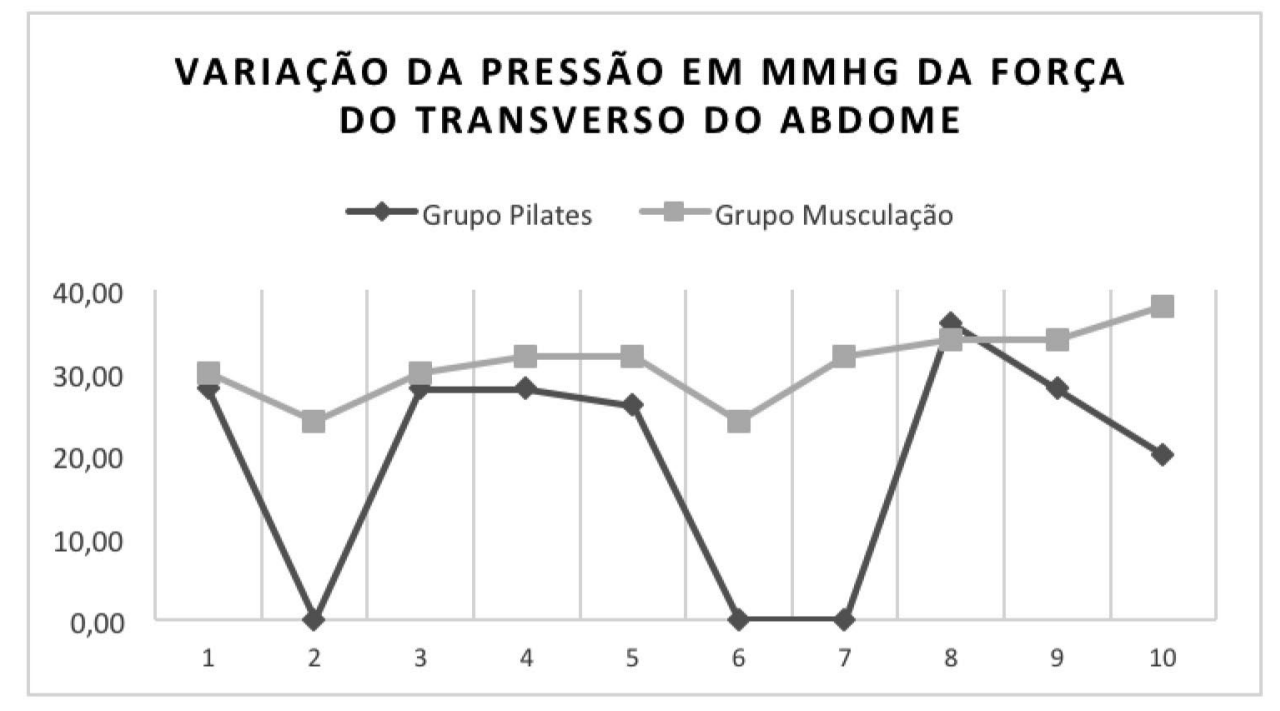

Abaixo na figura 2, representa os dados coletados do teste do sentar-alcançar, avaliando a flexibilidade em $\mathrm{cm}$ entre as praticantes do grupo Pilates e musculação.

Figura 2. Gráfico representando o desempenho da flexibilidade em (cm) entre os grupos Pilates e musculação através do teste sentar-alcançar

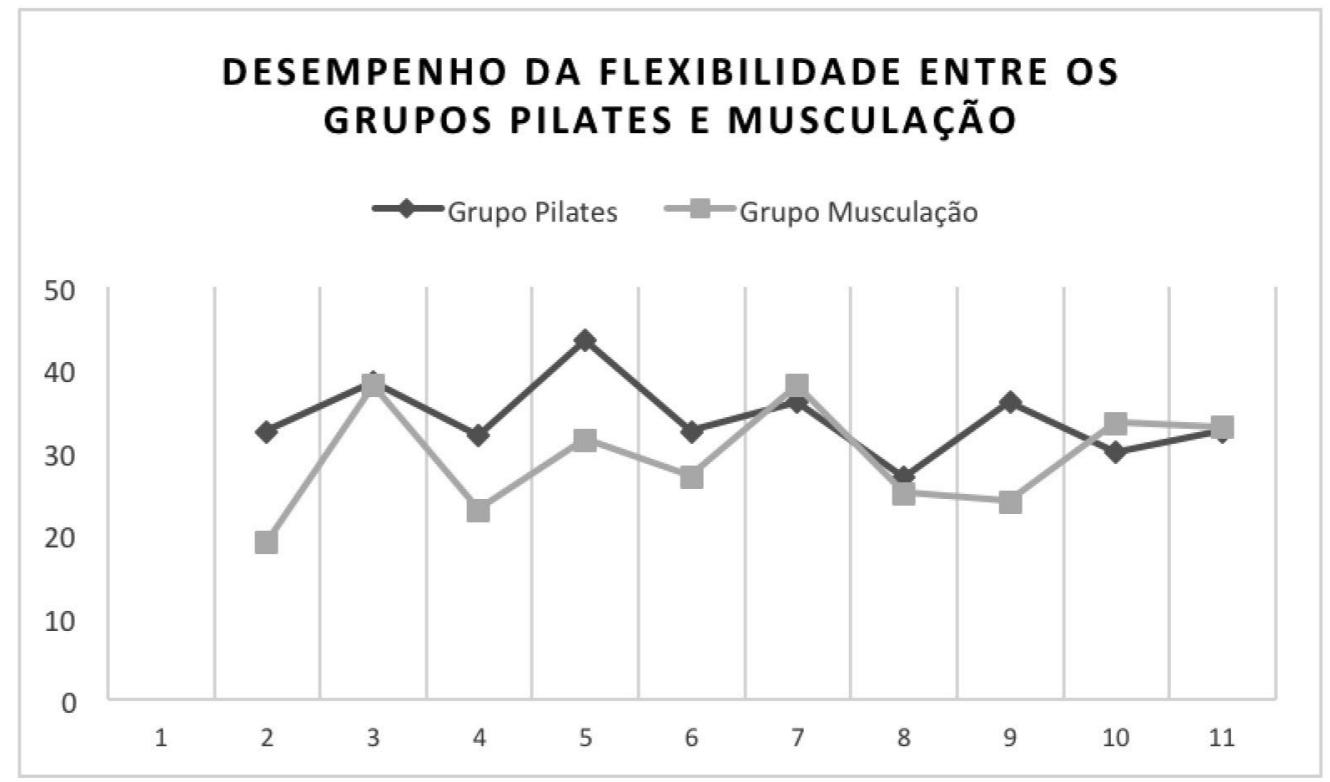

Na tabela 1, está representado o resumo estatístico descritivo dos resultados referentes ao teste esfigmomanômetro modificado nas praticantes do grupo Pilates e musculação.

Tabela 1. Resumo descritivo dos resultados do teste esfigmomanômetro modificado pelas praticantes dos grupos Pilates e musculação

\begin{tabular}{ccc}
\hline Domínios & Grupo Pilates & Grupo musculação \\
\hline Média & 19,40 & 31,00 \\
Mediana & 27,00 & 32,00 \\
Desvio padrão & 13,92 & 4,35 \\
Mínimo & - & 24,00 \\
Máximo & 36,00 & 38,00 \\
\hline
\end{tabular}


Na tabela 1 pode-se verificar que, quando avaliado a força muscular do transverso do abdome das integrantes de ambos os grupos, as praticantes do método Pilates apresentaram um aumento da pressão abdominal (em termos de diminuição de pressão por $\mathrm{mmHg}$ ) em média de 19,40 $\pm 13,92 \mathrm{mmHg}$, enquanto que as do grupo musculação obtiveram uma média de $31,00 \pm 4,35 \mathrm{mmHg}$.

Para a média do grupo Pilates, são considerados com força muscular normal os compreendidos entre 5,50 e $33,30 \mathrm{mmHg}$. Os abaixo deste primeiro apresentam um ótimo desempenho da musculatura enquanto os acima do segundo valor podemos considerar de mal desempenho. Os $50 \%$ da amostra encontram-se abaixo ou igual a $27 \mathrm{mmHg}$. Os valores abaixo representam a metade de melhor desempenho muscular. Em contrapartida, a média do grupo musculação são considerados com força muscular normal os compreendidos entre 26,70 e 35,30 $\mathrm{mmHg}$ e $50 \%$ da amostras encontram-se abaixo ou igual $32,00 \mathrm{mmHg}$.

Os valores mínimos de pressão (em termos de aumento de pressão abdominal) encontrados do grupo Pilates são de $0,00 \mathrm{mmHg}$ representado pelo símbolo (-) se mostrou superior em comparação ao grupo musculação: $24,00 \mathrm{mmHg}$.

A tabela 2, representa o resumo estatístico descritivo, referente ao teste TSA nas praticantes do grupo Pilates e musculação.

Tabela 2. Resumo descritivo dos resultados do teste sentar-alcançar apresentados pelas praticantes dos grupos Pilates e musculação

\begin{tabular}{ccc}
\hline Domínios & Grupo Pilates & Grupo musculação \\
\hline Média & 34,05 & 29,20 \\
Mediana & 32,50 & 29,25 \\
Desvio padrão & 4,65 & 6,54 \\
Mínimo & 27,00 & 19,00 \\
Máximo & 43,50 & 38,00 \\
\hline
\end{tabular}

Na tabela 2, quanto ao nível de flexibilidade dos dois grupos coletados, foi possível observar que o nível médio de flexibilidade das praticantes do grupo Pilates foi de $34,05 \pm 4,65 \mathrm{~cm}$, sendo que os valores normais considerados estão entre 30,50 e 39,10 cm. Os 50\% da amostra do grupo Pilates encontram-se acima ou igual a 32,50 cm representando a metade de melhor desempenho de flexibilidade. Em contrapartida, o grupo musculação apresentou média de $29,20 \pm 6,54 \mathrm{~cm}$ e os valores normais considerados então entre 22,70 e $35,70 \mathrm{~cm}$. Os $50 \%$ da amostra do grupo musculação encontram-se acima ou igual a $29,25 \mathrm{~cm}$, sendo considerado a metade de melhor desempenho de flexibilidade. Os valores mínimos e máximos encontrados para a flexibilidade do grupo Pilates foram de 27,00 e 43,50 cm respectivamente, em comparação ao grupo musculação são de 19,00 e 38,00 cm respectivamente.

A tabela 3, representa a comparação intergrupos dos resultados expressos em média e desvio-padrão do TEM e TSA por meio do teste T de student $\alpha=0,05$ para amostras independentes dos grupos Pilates e musculação.

Tabela 3. Comparação intergrupos dos resultados do TEM e TSA aplicados expressos em média e desvio-padrão (DP \pm ) por meio do teste T de student para as amostras independentes

\begin{tabular}{cccc}
\hline & Grupo Pilates & Grupo Musculação & $(p<0,05)$ \\
\hline TEM & $19,40 \pm 13,92$ & $31,00 \pm 4,35$ & 0,02 \\
TSA & $34,05 \pm 4,65$ & $29,20 \pm 6,54$ & 0,07 \\
\hline
\end{tabular}


$\mathrm{Na}$ tabela 3, onde as médias dos dois testes entre os dois grupos são comparados, para o TEM foi possível constatar os efeitos provocados como resposta aos específicos métodos de treinamento, com diferenças estatísticas em favor do grupo Pilates. Porém, no TSA, após a análise do teste T de student, não foi encontrado diferenças estatísticas significativas entre os dois grupos.

\section{Discussão}

Através da aplicação do teste T de student foi verificado valores significativos de superiores para a força muscular do transverso do abdome do grupo Pilates quando comparado ao grupo musculação. Nesse estudo foi possível observar que as praticantes do método Pilates obtiveram um melhor desempenho da musculatura do transverso do abdome com média de 19,40 mmHg em comparação ao grupo musculação que obteve a média de 31 . Sendo assim, quanto mais eficaz a contração, maior deveria ser a redução de pressão, sendo evidenciado a maior ativação do músculo (por menor pressão registrada no esfigmomanômetro) no grupo Pilates. Uma redução de pressão maior ou igual a $4 \mathrm{mmHg}$ é considerada uma resposta eficaz para indivíduos não-atletas, e de 2 a $4 \mathrm{mmHg}$ é considerada uma resposta média ${ }^{14}$. Portanto, ambos os grupos obtiveram uma resposta eficaz para a ativação do transverso do abdome, com exceção de uma praticante do grupo musculação que não obteve uma ativação muscular satisfatória. Segundo Ferreira et al. $\frac{15}{}$ realizaram um estudo com o objetivo de analisar a influência do método Pilates em 12 voluntárias adultas do sexo feminino, na qual enfatizam que o método Pilates exerce significativa estimulação na musculatura estabilizadora da coluna vertebral, a qual inclui a parede abdominal, além de melhorar o controle consciente de todos os movimentos musculares do corpo.

Constata-se que uma intervenção de exercícios de Pilates conveniente pode melhorar significativamente a força muscular e flexibilidade do tronco em muIheres ${ }^{16}$. Esse achado está de acordo com o encontrado nessa pesquisa, onde foi observado que mulheres praticantes do método pilates apresentam força muscular do músculo transverso do addomen com valores estatisticamente superiores quando comparadas ao grupo praticante de musculação.
Quanto a flexibilidade, na presente pesquisa observou-se uma melhor flexibilidade no grupo praticante de Pilates, embora não significativa o que corrobora com o estudo de Siqueira et al. $\stackrel{17}{ }$ que observou que em dez sessões de Pilates não foi possível ocorrer mudanças no trofismo abdominal e na flexibilidade do tronco. Foi possível observar que as praticantes do grupo Pilates obtiveram um desempenho médio de $34,05 \mathrm{~cm}$ e valor máximo de $43,50 \mathrm{~cm}$, sendo escores maiores quando comparados ao grupo de musculação que foram de $29,20 \mathrm{~cm}$ e valores máximos de $38,00 \mathrm{~cm}$. De acordo com a classificação segundo Canadian Standardizes Test of Fitness (CSTF) para as faixas etárias de 30 - 39 anos e 40 - 49 anos conforme idade da amostra do grupo, a média do grupo Pilates está classificado como acima da média e na média ${ }^{18}$. Para a média do grupo musculação, compreende a classificação abaixo da média. No estudo de Lima et al. .9 foram escolhidas vinte mulheres adultas sendo 10 delas praticantes de Pilates e 10 praticantes de musculação com o objetivo de analisar a influência do método Pilates e da musculação sobre a resistência da musculatura abdominal e flexibilidade em mulheres. $O$ estudo demonstrou que ambos os grupos foram beneficiados com o alcance de níveis adequados de capacidades motoras, embora, no presente estudo, entre os distintos métodos de treinamento, o método Pilates promoveu maior estímulo para a flexibilidade e resistência abdominal. Diversos estudos demonstraram que o método Pilates pode promover um aumento da amplitude no movimento articular ${ }^{17,18}$, o que gera a promoção de maiores níveis de flexibilidade do que outras práticas de atividades físicas, podendo ser uma das explicações para o método Pilates ter apresentado resultados superiores do que a musculação 9,10,15,16.

A instabilidade da coluna tem sido destacada como causa significativa de dor lombar e um fator causal em 20 a 30\% das doenças crônicas da coluna e a alteração no padrão de recrutamento dos músculos abdominais é um achado comum nos pacientes ${ }^{19}$. Estudos demonstram que indivíduos com lombalgia crônica apresentam disfunção da musculatura abdominal profunda, em especial o músculo transverso do abdome. Observaram também que este músculo em episódio de dor lombar apresenta diminuição de força, atrofia e atraso na velocidade de disparo ${ }^{20}$. O método Pilates demonstra ser uma alternativa de tratamento para melhora da flexibilidade e força e redução da dor lombar ${ }^{21}$. Diante disto, fica evidente a importância do musculo transverso 
do abdome para gerar estabilidade e redução das patologias lombares 22 .

Algumas evidências cientificas relatam que o método Pilates embora não apresente efeitos significativos no alivio da dor lombar crônica, pode ser mais benéfico que outras modalidades de fisioterapia ${ }^{23,24}$. Entretanto, de acordo com estudos realizados fica evidente que o método Pilates reduz a dor lombar independentemente de sua natureza além de promover uma melhora na qualidade de vida e aumento da flexibilidade $20,23, \underline{25}$.

\section{Conclusão}

Através deste estudo pode-se concluir que mulheres praticantes do método Pilates apresentaram força muscular de transverso de abdome superiores as que praticam musculação. Já em relação a flexibilidade, embora não encontrado valores estatisticamente significativos entre os grupos, existe uma tendência de meIhor desempenho também nas praticantes de Pilates de acordo com Canadian Standardizes Test of Fitness.

\section{Contribuições dos autores}

Pereira Júnior AA e Silva A participaram da concepção, delineamento, orientação da análise estatística dos dados da pesquisa e interpretação dos resultados, e redação do artigo científico.

\section{Conflitos de interesses}

Nenhum conflito financeiro, legal ou político envolvendo terceiros (governo, empresas e fundações privadas, etc.) foi declarado para nenhum aspecto do trabalho submetido (incluindo, mas não se limitando a subvenções e financiamentos, participação em conselho consultivo, desenho de estudo, preparação de manuscrito, análise estatística, etc.).

\section{Referências}

1. Caputo EL, Costa MZ. Influence of physical activity on quality of life in postmenopausal women with osteoporosis. Rev Bras Reumatolog. 2014;54(6):467-473. doi: 10.1016/j.rbr.2014.02.008
2. Lara S, Wendt P, Silva ML. Comparação da qualidade de vida em mulheres praticantes de Pilates e musculação. ConScientiae Saúde. 2014;13(1):134-140. doi: 10.5585/conssaude.v13n1.4607

3. Anjos MCG, Pimentel JPD, Epifânio DS, Malheiro A, Passos, LFS, Costa AG. The effects of brief physical conditioning on imune cells and cytokines in elderly individuals in Manaus, Amazonas. Fisioter Mov. 2016;29(2):305-315. doi: 10.1590/0103-5150.029.002.A009

4. Lima AP, Silva ASD, Cardoso FB. Comparação dos níveis de flexibilidade de mulheres praticantes de hidroginástica e Pilates. ConScientiae Saúde. 2015;14(3):363-369. doi: 10.5585/conssaude. $\underline{\mathrm{v} 14 \mathrm{n} 3.4981}$

5. Morais PAO, Dantas MGB, Pinotti M, Pitangui ACR, Araújo RC. Influência de diferentes calçados na postura e na atividade dos músculos do tronco. ConScientiae Saúde. 2012; 11(3):462-469. doi: $10.5585 /$ conssaude.v11n3.3340

6. Costa LOP, Costa LCM, Cançado RL, Oliveira WM, Ferreira $\mathrm{PH}$. Confiabilidade do teste palpatório e do uso do músculo transverso abdominal em indivíduos normais. Acta Fisiátr. 2004;11(3):101-105.

7. Kisner C, Colby LA. Exercícios terapêuticos: fundamentos e técnicas. Manole; 2005.

8. SchiessI M, Lima MCAM. Efeito de diferentes tempos de alongamento na flexibilidade de bailarinas. ConScientiae Saúde. 2015;14(3):456-462. doi: 10.5585/conssaude.v14n3.5464

9. Lima KA, Silva RM, Santos RM, Leite LM, Araújo SS. Efeitos da prática do método Pilates ${ }^{\circledR}$ e musculação sobre a aptidão física e composição corporal em mulheres. Biológicas e Saúde. 2011;1(1). doi: $10.25242 / 8868112011514$

10. Byrnes K, Wu PJ, Whillier S. Is Pilates an Effective Rehabilitation Tool? A Systematic Review. J Bodyw Mov Ther. 2018;22(1):192-202. doi: 10.1016/j.jbmt.2017.04.008

11. Oliveira DS, Ruffo AM. Exercício resistido: importância para a terceira idade. Uningá Review. 2012; 12(2):68-75.

12. Souza LAC, Martins JC, Salmela LFT, Godoy MR, Aguiar LT, Faria CDCM. Avaliação da força muscular pelo teste do esfigmomanômetro modificado: uma revisão de literatura. Fisioter Mov. 2013; 26(2):437-52.

13. Bezerra ES, Martins SL, Leite TB, Paladino KDV, Rossato M, Simão R. Influência da modificação do teste de sentar e alcançar sobre o indicador de flexibilidade em diferentes faixas etárias. Motri. 2015;11(3):3-10. doi: 10.6063/motricidade.2336

14. Ceccato J, Varrialle M, Donadio MVF, Kiefer T, Vaz MA. Avaliação funcional do transverso do abdômen em remadores. Brazilian Journal of Biomechanics. 2011;12(22):9-13. 
15. Ferreira CB, Aidar FJ, Novaes GS, Vianna JM, Carneiro AL, Menezes LS. O método Pilates sobre a resistência muscular localizada em mulheres adultas. Motri. 2007;3(4):76-81.

16. Kao YH, Liou TH, Tsai YW, Wang KM, Huang YC. Effects of a 12-Week Pilates Course on Lower Limb Muscle Strength and Trunk Flexibility in Women Living in the Community. Health Care for Women International. 2015;36(3):303-319. doi: 10.1080/07399332.2014.900062

17. Siqueira GR, Alencar GG, Oliveira ECM, Teixeira VQM. Efeito do Pilates sobre a flexibilidade do tronco e as medidas ultrassonográficas dos músculos abdominais. Rev Bras Med Esporte. 2015;21(2):139-143. doi: 10.1590/1517$\underline{86922015210202180}$

18. Ribeiro CCA, Abad CCC, Barros RV, Barros Neto TL. Nível de flexibilidade obtida pelo teste de sentar e alcançar a partir de estudo realizado na Grande São Paulo. Rev Bras Cineantropom Desempenho Hum. 2010;12(6):415-421. doi: 10.1590/S198000372010000600004

19. Cadó T, Ataualpa A, Schuster D, Cabral J, Braz M, Zambarda S et al. Avaliação da ativação dos músculos do core de acadêmicos do curso de fisioterapia. Revista Contexto \& Saúde. 2011;11(20):737-742. doi: 10.21527/2176-7114.2011.20.737-742
20. Ramos LAV. Avaliação da fadiga do músculo multífido lombar e ativação do transverso do abdome em indivíduos com hérnia discal lombar [dissertação]. São Paulo: Faculdade de Medicina da Universidade de São Paulo; 2012.

21. Sinzato CR, Taciro C, Pio CA, Toledo AM, Cardoso JR, Carregaro RL. Efeitos de 20 sessões do método Pilates no alinhamento postural e flexibilidade de mulheres jovens: estudo piloto. Fisioter Pesq. 2013;20(2):143-150. doi: 10.1590/S1809$\underline{29502013000200008}$

22. Gonçalves MBK, Angelo RCO, Martins PPC. Aspectos clínicos e morfofuncionais da casa de força no método Pilates. Fisioter Bras. 2009;10(1):54-58. doi: 10.33233/fb.v10i1.1500

23. Patti A, Bianco A, Paoli A, Messina G, Montalto MA, Bellafiore $M$ et al. Effects of pilates exercise programs in people with chronic low back pain. Medicine (Baltimore). 2015;94(4):e383. doi: 10.1097/MD.0000000000000383

24. Yamato TP, Maher CG, Saragiotto BT, Hancock MJ, Ostelo RWJG, Cabral CMN et al. Pilates for low back pain. Cochrane Database Syst Rev. 2015;(7):CD010265. doi: 10.1002/14651858. CD010265.pub2

25. Wasser JG, Vasilopoulos T, Zdziarski LA, Vincent HK. Exercise benefits for chronic low back pain in overweight and obese individuals. PM\&R. 2017;9(2):181-192. doi: 10.1016/j. pmrj.2016.06.019 